\title{
FATORES CRÍTICOS PARA A DISSEMINAÇÃO DA CULTURA DA INOVAÇÃO ATRAVÉS DA GESTÃO DO CONHECIMENTO
}

\section{CRITICAL FACTORS FOR THE DISSEMINATION OF THE CULTURE OF INNOVATION THROUGH KNOWLEDGE MANAGEMENT}

\author{
Glória Maria Perez de Moura ${ }^{1}$; Marcos Gilson Gomes Feitosa ${ }^{2}$; Fabiana Ferreira Silva ${ }^{3}$; \\ Flávia Andreza de Souza ${ }^{4}$ \\ ${ }^{1}$ Serviço Nacional de Aprendizagem Industrial - SENAI - Recife/PE - Brasil \\ gperez@pe.senai.br \\ ${ }^{2}$ Universidade Federal de Pernambuco - UFPE - Recife/PE - Brasil \\ feitosam@terra.com.br \\ ${ }^{3}$ Faculdade Santa Cruz - FACRUZ - Santa Cruz do Capibaribe/PE - Brasil \\ professorafabyana@hotmail.com \\ ${ }^{4}$ Faculdades Integradas do Sertão - FIS - Serra Talhada/PE - Brasil \\ fappita@hotmail.com
}

\begin{abstract}
Resumo
A capacidade de inovar é atualmente considerada uma das mais importantes características das organizações de excelência. Neste contexto, percebe-se a cultura como um elemento essencial ao desenvolvimento da inovação e da gestão do conhecimento em uma empresa, podendo influir de forma positiva ou negativa nesse processo. Diante do exposto, a questão que norteou o desenvolvimento deste trabalho foi a seguinte: "Quais seriam as ações e práticas que facilitam ou dificultam o desenvolvimento de uma cultura voltada à inovação e à gestão do conhecimento no SENAI Pernambuco?" A fundamentação teórico-empírica do estudo teve como base textos e pesquisas sobre gestão do conhecimento, inovação e cultura organizacional. A pesquisa caracteriza-se como um estudo qualitativo, exploratório e descritivo, através de um estudo de caso único. A técnica de coleta de dados utilizada consistiu na aplicação de um questionário aberto enviado para todos os colaboradores da instituição, permitindo obter dados significativos a respeito da cultura organizacional. Dentre os resultados alcançados, ressalta-se como ação facilitadora "investimentos em educação e desenvolvimento de talentos humanos" e como ação dificultadora o "fator tempo", tanto para o diálogo como para a elaboração de projetos $e$ pesquisas na instituição. Como ações a serem desenvolvidas, constatamos a importância de a instituição orientar, capacitar e fomentar a pesquisa, acompanhar, organizar e disseminar a produção intelectual dos colaboradores (monografias, dissertações, projetos), aperfeiçoar a gestão da informação através de meios eletrônicos, criar um banco de talentos humanos para apoiar a gestão por competências e criar uma política de gestão do conhecimento.
\end{abstract}

Palavras-chave: cultura organizacional; inovação; gestão do conhecimento. 


\section{Introdução}

A complexidade no mundo do trabalho impulsionada pela globalização da economia leva as organizações a focar em setores cujos processos e/ou produtos são cada vez mais intensivos em tecnologia de informação, conhecimento e inovação.

Ao transportarmos essa temática para instituições de ensino, percebemos a relevância da Gestão do Conhecimento neste tipo de organização e, mais ainda, ao analisarmos uma instituição de ensino profissionalizante como o SENAI Pernambuco cujo escopo de atuação tem como base a aprendizagem e a inovação. A relevância desse estudo justifica-se, inicialmente, porque o Plano Estratégico do SENAI-PE, do período de 2008 a 2010, destaca como um dos objetivos principais "o desenvolvimento de uma cultura voltada à inovação e à gestão do conhecimento". No âmbito acadêmico, este trabalho vem cumprir um dos papéis sociais da universidade a partir da aplicabilidade da pesquisa científica a uma instituição de conhecimento de referência nacional, tendo a sociedade como maior beneficiária de modo geral.

Nesse contexto, a questão norteadora do estudo foi: Quais seriam as ações e práticas que facilitam ou dificultam o desenvolvimento de uma cultura voltada à inovação e à gestão do conhecimento no SENAI Pernambuco? Diante do exposto, o objetivo geral desta pesquisa foi identificar as ações e práticas que facilitam ou dificultam o desenvolvimento de uma cultura voltada à inovação e à gestão do conhecimento no SENAI Pernambuco. Para tanto, foram elaborados os seguintes objetivos específicos:

a) Entender a relação entre as teorias e conceitos que abordam cultura, inovação e gestão do conhecimento;

b) Diagnosticar as ações e práticas percebidas pelos colaboradores do SENAI-PE, que podem facilitar ou dificultar o desenvolvimento de uma cultura voltada à inovação e a gestão do conhecimento;

c) Verificar que ações e práticas o SENAI-PE poderia implementar encorajando os colaboradores a colocar em prática suas ideias.

Para tanto, este ensaio teórico-empírico está dividido em cinco seções: esta primeira que contextualiza o tema e a problemática investigada; a segunda que traz a literatura que fundamentou a realização da pesquisa com ênfase na gestão do conhecimento e da inovação; a terceira seção contempla os procedimentos metodológicos utilizados; a quarta seção traz os resultados da pesquisa; e, por fim, são apresentadas considerações finais e referências bibliográficas.

\section{Referencial Teórico}

Gestão do Conhecimento, Inovação e Cultura Organizacional constituem os três pilares que serviram de base para o desenvolvimento desta pesquisa teórico-empírica, conforme apresentado de 
forma mais detalhada a seguir.

\subsection{A gestão do conhecimento como diferencial de excelência nas organizações}

A importância do conhecimento para o desenvolvimento de uma organização sempre existiu desde os primórdios das teorias organizacionais baseadas no pensamento administrativo. Mais recentemente este tema tornou-se evidente devido a estarmos vivendo na sociedade do conhecimento. "A Gestão do Conhecimento pode ser definida como um processo, articulado e intencional, destinado a sustentar ou a promover o desempenho global de uma organização, tendo como base a criação e a circulação de conhecimento" (SALIM, 2001 apud CANONGIA et al, 2004).

As áreas de conhecimento que constituem a gestão do conhecimento são a gestão da informação, compreendendo as áreas de tecnologia e ciência da informação, para a construção de conhecimento codificado e a gestão de pessoas voltada para o entendimento da dinâmica dos processos de criação e disseminação de conhecimento (SVEIBY, 2002 apud CANONGIA et al, 2004). Estes autores caracterizam o processo de gestão do conhecimento com foco em duas perspectivas: a perspectiva individual que analisa as motivações e as capacidades do indivíduo e a perspectiva organizacional que estabelece os recursos e as competências essenciais às organizações. Pesquisas realizadas na última década destacam a integração entre desempenho da organização e gestão de talentos humanos. O capital humano torna-se componente crítico para o sucesso das organizações (NOVELLI, 2003). Sua importância torna-se reconhecida e sua contribuição pode ser evidenciada quando existe o aumento da competitividade decorrente de inovações tecnológicas e organizacionais.

Newell (2005) diz que o conhecimento pode ser visto sob duas perspectivas: o conhecimento como posse, isto é o conhecimento pode ser explicitado e transferido de uma pessoa para outra, grupo ou organização; e o conhecimento como um fenômeno construído socialmente. Nessa perspectiva, o conhecimento se dá na pratica, através da interação e do diálogo entre as pessoas. Parte do conhecimento da organização é explícito e formal e parte é implícito e informal, gerado a partir da prática. E, por estar integrado à pratica, o conhecimento organizacional é disperso e ambíguo. É disperso porque só existe a partir da interação entre as pessoas e é ambíguo porque cada indivíduo vê e interpreta os processos de uma forma particular.

Desta forma, a criação do conhecimento é resultado da interação entre as pessoas e essa interação social é chamada de conversão do conhecimento. Para Nonaka e Takeuchi (1997), o conhecimento é dividido em quatro tipos de conversão:

a) Na socialização os indivíduos compartilham o conhecimento tácito através da prática ou observação, isto é, através de trocas de experiências em grupo (de tácito para tácito); 
b) A externalização é a conversão do conhecimento tácito em conhecimento explícito e sua comunicação ao grupo, é realizada através de experiências e percepções das pessoas. É nesse momento que se dá a transferência de conhecimentos (de tácito para explicito) e se dá a criação de novos conceitos;

c) A combinação refere-se à sistematização do conhecimento, onde acontece a organização e o registro dos conhecimentos, em novos agrupamentos (de explícito para explícito); d) E a internalização que constitui o processo através do qual são desenvolvidos novos conhecimentos tácitos e novos modelos mentais (de explícito para tácito). A criação e compartilhamento do conhecimento nas organizações provêm não somente das pessoas, mas também das condições e estruturas oferecidas pelas empresas. Existem oito fatores que são estratégicos para a implantação da gestão do conhecimento na organização. São eles:

Quadro 1 - Fatores estratégicos para a implantação da gestão do conhecimento na organização.

\begin{tabular}{|l|l|}
\hline \multicolumn{1}{|c|}{ Fatores } & \multicolumn{1}{c|}{ Descrição } \\
\hline Alta Administração & $\begin{array}{l}\text { Ações e decisões administrativas no suporte ao desenvolvimento e gestão do } \\
\text { conhecimento. }\end{array}$ \\
\hline Cultura Organizacional & $\begin{array}{l}\text { Aspectos da cultura influindo positivamente no processo de gestão do } \\
\text { conhecimento. }\end{array}$ \\
\hline Novas Estruturas Organizacionais & Nova visão organizacional com equipes multidisciplinares. \\
\hline $\begin{array}{l}\text { Práticas e Políticas de Gestão de } \\
\text { Pessoas }\end{array}$ & $\begin{array}{l}\text { Aspectos ligados à contratação, qualificação e gestão das pessoas, na agregação } \\
\text { do conhecimento. }\end{array}$ \\
\hline $\begin{array}{l}\text { Tecnologias e Sistemas de } \\
\text { Informação }\end{array}$ & Políticas internas de acesso a informação. \\
\hline Mensuração de Resultados & $\begin{array}{l}\text { Necessidades de perceber os investimentos em projetos de gestão do } \\
\text { conhecimento. }\end{array}$ \\
\hline Aprendizado com o Ambiente & $\begin{array}{l}\text { A influência de variáveis externas e elementos do ambiente externo que } \\
\text { interferem na formação do conhecimento organizacional. }\end{array}$ \\
\hline Orientação para Processos & Ações voltadas à organização interna processual. \\
\hline
\end{tabular}

Fonte: Adaptado de Davenport e Prusak (1998 apud FURLANETTO et al, 2006) e Terra (2005 apud FURLANETTO et al, 2006)

Dessa forma, percebe-se que o sucesso da gestão do conhecimento depende não somente da vontade do indivíduo de querer aprender e compartilhar o seu conhecimento, mas principalmente de todo um conjunto de fatores estratégicos da organização, como diretrizes, sistemas, estruturas e, principalmente, de uma cultura que propicie um ambiente adequado à implantação do processo de gestão do conhecimento.

Robbins (2005) ressalta a importância da gestão do conhecimento para as organizações e revela que ela está pautada em três motivos: o primeiro é que as empresas que sabem utilizar bem os conhecimentos e experiências de seus talentos humanos alcançam melhores resultados entre seus concorrentes; o segundo é a consciência de algumas empresas perceberem a necessidade de criar mecanismos de retenção dos conhecimentos de colaboradores talentosos, antes que eles deixem a organização; e o terceiro motivo é que se a empresa tem um bom sistema de gestão do 
conhecimento, ao iniciar um novo projeto, ela não precisa começar do zero, as experiências e os conhecimentos adquiridos e armazenados por outros colaboradores darão as informações necessárias para a continuação desse trabalho, eliminando o retrabalho e tornando a organização mais eficiente. $\mathrm{O}$ autor ainda enfatiza que os conhecimentos e habilidades dos colaboradores devem ser organizados num banco de dados informatizado e que se criem mecanismos de fácil acesso a essas informações, além da necessidade de se criar uma cultura que encoraje e apóie o compartilhamento de conhecimentos, estabelecendo formas de socialização entre os colaboradores da organização.

O constructo aprendizagem está intimamente relacionado à Gestão do Conhecimento. Para Robbins (2005), a aprendizagem só acontece quando existe mudança de comportamento. Já o termo mudança no contexto organizacional remete à idéia de novas práticas, ou seja, inovação em produtos, processos e até mesmo na gestão. Portanto, apresentamos a seguir o papel da inovação no contexto organizacional.

\subsection{A importância da inovação para as organizações}

A geração, a exploração e a difusão do conhecimento são fundamentais para o crescimento econômico, o desenvolvimento e o bem-estar das nações. Compreende-se, de forma geral, que a inovação seja um aspecto essencial à sustentabilidade das organizações. As inovações organizacionais podem ter um impacto importante sobre o desempenho da sociedade, em especial, mediados pelas empresas. Inovações organizacionais podem melhorar a qualidade e a eficiência do trabalho, acentuar a troca de informações e aperfeiçoar a competência organizacional para aprender, sistematizar e também criar novos conhecimentos.

Tomaél et al (2005) evidenciam a necessidade da constante acumulação de conhecimentos e contínua capacidade tecnológica. Destaca-se então a aprendizagem organizacional, apresentando-se como um relevante processo para o desenvolvimento da inovação tecnológica. Entende-se a aprendizagem organizacional como um processo que deve ser construído em longo prazo no qual as pessoas aprendem em grupo, refletindo sobre suas experiências passadas com a finalidade de orientar ações futuras.

As inovações organizacionais podem referir-se à implementação de novos métodos, tais como mudanças em práticas de negócios, na organização do local de trabalho, nas relações externas da empresa, no aprimoramento da capacidade de inovação da empresa: aumentando sua capacidade para desenvolver novos produtos, processos, ou ainda, para ganhar e criar novos conhecimentos.

Segundo a Organização para Cooperação e Desenvolvimento Econômico, as empresas inovam para a melhoria de seu desempenho, aumento da produtividade, maior vantagem competitiva, buscando elevar a eficiência e a qualidade de suas operações. Por exemplo, 
melhoramentos nos processos de produção podem permitir o desenvolvimento de um novo leque de produtos; ao mesmo tempo que novas práticas organizacionais podem melhorar a capacidade empresarial de adquirir e criar novos conhecimentos que poderão ser usados para o desenvolvimento de outras inovações (OCDE, 1997).

A inovação requer a utilização do conhecimento novo ou um novo uso ou combinação para o conhecimento existente. O conhecimento novo pode ser gerado pela empresa inovadora no curso de suas atividades ou adquirido externamente. $\mathrm{O}$ uso de conhecimento novo ou a combinação do conhecimento existente requerem esforços inovadores que se distinguem das rotinas padronizadas. Conforme Canongia (2004) a capacidade de inovar é uma das mais fortes características das organizações competitivas. A busca constante por inovações radicais, aquelas capazes de criar novos mercados e por inovações incrementais, isto é, aquelas que estão sempre buscando a melhoria dos processos, são imprescindíveis para as organizações. A capacidade de inovar é atualmente considerada uma das mais importantes características das organizações de excelência, no entanto, as empresas precisam promover um ambiente de estímulo à inovação. Segundo Barbieri e Álvares (2004 apud ANDREASSI, 2007, p. 01) "o verbo inovar vem do latim (innovare) e significa renovar ou introduzir novidades de qualquer espécie. Já inovação é uma variante e quer dizer renovado ou tornado novo".

A geração de idéias novas é essencialmente uma questão individual e/ou de pequenos grupos. No entanto, a inovação torna-se um processo coletivo uma vez que altera crenças, hábitos e interesses sedimentados em indivíduos e grupos. A inovação pode alterar significativamente produtos, serviços, ações e valores da organização. Segundo Stevens (2011) o processo de inovação em uma organização deve seguir cinco princípios:

Quadro 2-Cinco princípios do processo de inovação

\begin{tabular}{|l|l|}
\hline Princípios & \multicolumn{1}{c|}{ Descrição } \\
\hline PRIMEIRO & $\begin{array}{l}\text { A inovação deve ser abordada como disciplina. Para praticar a inovação como uma disciplina é } \\
\text { necessário, primeiro, distinguir criatividade (levantamento de idéias) de inovação, isto é, o processo } \\
\text { de trazer para a organização bons resultados. Algumas organizações costumam promover a } \\
\text { criatividade através de sessões de brainstorning, entretanto, na maioria das vezes, apesar de terem } \\
\text { surgido ótimas idéias, elas não saem do papel, ficando massacradas pelas pressões do cotidiano. }\end{array}$ \\
\hline SEGUNDO & $\begin{array}{l}\text { Inovação deve ser abordada de forma inclusiva. Ela não deve se restringir a um único departamento, } \\
\text { deve tornar-se parte do DNA de toda a organização. }\end{array}$ \\
\hline TERCEIRO & $\begin{array}{l}\text { Inovação deve incluir uma sistemática e permanente pesquisa para novas oportunidades. } \\
\text { QUARTO quãão deve envolver todos na organização. Atualmente, na maioria das organizações, novas idéias } \\
\text { base pensem e tomem decisões. Portanto, não procuram estimular e levantar as boas idéias de seu } \\
\text { quadro de pessoal. A mudança desse paradigma, ou seja, a descentralização do monopólio e controle das } \\
\text { idéias, será um dos grandes desafios do século XXI. }\end{array}$ \\
\hline QUINTO & $\begin{array}{l}\text { A Inovação deve ser centrada no cliente. Os clientes contemporâneos, por deterem mais } \\
\text { informações, são mais exigentes e a disciplina da inovação significa entre outras coisas ouvir os } \\
\text { clientes de novas maneiras. Significa oferecer produtos de melhor qualidade, buscar sempre o } \\
\text { aperfeiçoamento dos serviços e verificar se aquelas novas idéias estão efetivamente impulsionando o } \\
\text { crescimento das organizações. }\end{array}$ \\
\hline
\end{tabular}


Portanto, a inovação deve ser considerada como um processo sistêmico e permanente nas organizações. Por outro lado, Pèrez et al (2004 apud GUIMARÃES e SILVA, 2008) sugerem que a inovação em contextos organizacionais depende também da intenção em adotar algo considerado novo na empresa. Assim, a partir da intenção em inovar a organização mobilizará recursos para alcançar resultados positivos viabilizados pelo processo de pré-disposição à aprendizagem.

Esta nova abordagem traz a relevância de um ambiente saudável onde haja confiança no processo de comunicação, fazendo-nos refletir sobre a importância da Teoria da Ação Comunicativa, voltada para o entendimento, que é defendida por Habermas (2002). Uma cultura organizacional baseada no diálogo e na participação dos colaboradores poderá propiciar um entendimento recíproco, além da troca de informações e conhecimentos.

\subsection{Cultura Organizacional}

A cultura constitui um dos fatores estratégicos de maior importância para a implantação da gestão do conhecimento e da inovação em uma organização, podendo influir de forma positiva ou negativa nesse processo. A existência de uma cultura interna onde os colaboradores tenham clareza dos objetivos organizacionais, valorizem o trabalho em equipe e o compartilhamento de ideias, contribui para a gestão do conhecimento e para a inovação. Por outro lado, se a cultura é constituída de crenças, valores e costumes pautados em práticas passivas e cômodas, prejudica a criação de ideias e projetos inovadores no ambiente de trabalho.

Alves (1997) entende que a cultura da empresa é representada por um sistema complexo de crenças, valores, pressupostos, normas, símbolos, conhecimentos e significados. A cultura traduz ou reflete as escolhas e preferências que foram desenvolvidas pela liderança, atual ou passada, e que são compartilhadas pelos seus demais membros. Para este autor, uma das maneiras de apreciar a natureza da cultura organizacional é observando o funcionamento do dia-a-dia da empresa. As características de uma cultura que estão sendo observadas tornar-se-ão evidenciadas, à medida que se conheçam os padrões de interação entre os indivíduos, a linguagem que é utilizada, as imagens e temas explorados na conversa, bem como os vários rituais da rotina diária. À medida que se explora o que é racional dos aspectos desta cultura, pode-se quase sempre descobrir que existem explicações históricas para a maneira pelas quais as coisas são feitas.

Neste contexto, é fundamental entendermos o constructo percepção, uma vez que se trata da lente através da qual o observador vai tirar suas conclusões. Conforme Robbins (2005), percepção é o processo pelo qual os indivíduos organizam e interpretam suas impressões sensoriais com a finalidade de dar sentido ao seu ambiente. O mundo importante para o comportamento é o mundo na forma em que é percebido. Refere-se a construção e ao entendimento do mundo social a partir 
dos dados obtidos por meio dos sentidos. A percepção social refere-se aos processos pelos quais formamos nossas impressões das características e da personalidade das outras pessoas. Neste trabalho, procuraremos identificar as ações e práticas percebidas pelos colaboradores do SENAIPE, que podem facilitar ou dificultar o desenvolvimento de uma cultura voltada à inovação e à gestão do conhecimento. Souza et al (2008) enfatizam que estudos recentes realizados no Brasil mostram uma lacuna significativa no desenvolvimento da inovação de uma maneira geral nas organizações e de investimentos em pesquisa e desenvolvimento (P\&D). Considera que uma possível resposta para essa lacuna seria a falta de uma dimensão da inovação na cultura empresarial brasileira.

Isto confirma a importância de pensar a cultura, como uma construção social, dinâmica, realizada a partir da história das relações dos grupos sociais, em espaços e tempos distintos, não integrados totalmente. Gerar e compartilhar o conhecimento são manifestações culturais, provenientes de pessoas autônomas, emancipadas, que têm objetivos claros para sua vida, que buscam inovar, são corajosas e se permitem viver experiências e realizar práticas inovadoras. A adoção e a aplicação do conhecimento novo pode ser um processo lento e árduo, e o índice de sucesso será altamente influenciado por fatores culturais e estruturais da empresa em que o sistema será implementado (MUSSI e ANGELONI, 2002). É importante haver na organização uma cultura que incentive e possibilite o compartilhamento de informações e conhecimentos.

Tomaél et al (2005) destacam alguns aspectos para que o compartilhamento da informação e conhecimento obtenha sucesso: uma linguagem e uma cultura comum, sem as quais as pessoas não se entenderão nem tão pouco confiarão umas nas outras; a necessidade do contato face a face; e o status do possuidor do conhecimento que pode ou não inspirar confiança no conhecimento e na informação partilhada. A sistematização teórica acerca da Gestão do Conhecimento e da Inovação, tendo como plano de fundo, a Cultura Organizacional serviu de alicerce para alinhar o problema da pesquisa com a análise organizacional. A abordagem desses conceitos foi essencial à compreensão dos resultados empíricos obtidos, os quais são expostos nas próximas seções, relacionando a literatura pesquisada com a realidade apresentada pelos respondentes.

\subsection{Interface entre Inovação, Cultura e Gestão do Conhecimento}

A cultura de uma organização pode facilitar ou dificultar o acesso ao conhecimento. É importante se criar uma cultura que encoraje e apóie o compartilhamento de informações e conhecimentos, estabelecendo formas de socialização entre os atores da organização.

Gerentes talentosos, com entusiasmo e comprometidos com o trabalho de gestão do conhecimento são fundamentais para o alcance de resultados satisfatórios. A criatividade aumenta e inovações acabam surgindo como resultado da aplicação apropriada do conhecimento existente e da geração de novas ideias nos diversos tipos de organizações. No ensaio teórico sobre 
“Conhecimento, Aprendizagem e Inovação: uma proposta de articulação conceitual”, Guimarães e Silva (2008), destacam a importância da aprendizagem através da exteriorização do conhecimento tendo como resultado uma prática inovadora

É essencial desenvolver uma capacidade de inovação para o crescimento contínuo das organizações. Descobrir formas de criar um ambiente propício à descoberta, à geração de ideias e novos insights buscando envolver, interna e externamente o maior número possível de pessoas.

Pèrez et al (2004 apud GUIMARÃES e SILVA, 2008) sugerem que a inovação em contextos organizacionais depende também da intenção em adotar algo considerado novo na empresa. Percebe-se que a inovação só é disseminada em um ambiente se houver prontidão organizacional, espírito de colaboração e capacidade de absorção. Essas três competências organizacionais são imprescindíveis à implementação de atividades inovativas e relacionam-se diretamente com a cultura do local (HAFKESBRINK e SCHROLL, 2010, grifos nossos).

Portanto, a cultura é a base sobre a qual se desenvolve a gestão do conhecimento e esta é suporte para a inovação.

\section{Procedimentos Metodológicos}

Esta pesquisa tem caráter qualitativo, uma vez que busca compreender quais seriam as ações e práticas que facilitam ou dificultam o desenvolvimento de uma cultura voltada à inovação e à gestão do conhecimento no SENAI Pernambuco. Segundo Richardson (1989), os estudos que empregam uma metodologia qualitativa podem descrever a complexidade de determinado problema, analisar a interação de certas variáveis, compreender e classificar processos dinâmicos vividos por grupos sociais, contribuir no processo de mudanças de determinado grupo e possibilitar, em maior nível de profundidade, o entendimento das particularidades do comportamento dos indivíduos.

Trata-se de um estudo de caso, pois foi realizado com base na realidade de uma instituição e permitiu o estudo do fenômeno em profundidade dentro de um contexto bem delimitado (ROESCH, 1999). Este trabalho caracteriza-se ainda como um estudo exploratório e descritivo, uma vez que buscou o levantamento de informações sobre o problema apresentado.

A unidade analisada foi a instituição SENAI Pernambuco, composta por 616 colaboradores. A escolha desta instituição deveu-se à acessibilidade das pesquisadoras para realizar a pesquisa em consonância com o interesse da organização, uma vez que a questão de pesquisa está alinhada com um dos objetivos estratégicos da organização. Segundo Stake (1995) aspectos como tempo, acessibilidade e possibilidade para apreender as informações relevantes para o caso constituem os critérios essenciais para a seleção da unidade de análise, das técnicas e pessoas envolvidas. Entretanto, os critérios não se restringem apenas à conveniência para coleta de dados. Destacamos também a relevância teórico-empírica, haja visto que a unidade de análise é uma instituição de 
conhecimento e desenvolve um papel fundamental na sociedade quanto à geração e disseminação do conhecimento e da inovação.

Para estudar quais são as ações e práticas que facilitam ou dificultam o desenvolvimento de uma cultura voltada à inovação e à gestão do conhecimento no SENAI Pernambuco optou-se por envolver todos os cargos da instituição. A técnica de coleta de dados utilizada foi o questionário de pesquisa. Esse instrumento foi escolhido devido à limitação temporal para que fosse realizada a triangulação, não sendo possível realizar entrevistas e observações. Entretanto, foi enviado o questionário para todos os colaboradores da instituição (616 pessoas) e obtivemos um retorno de 329 respondentes, correspondente a 53\% das pessoas envolvidas na unidade de análise, não havendo distinção de cargos e de unidades, visto que o questionário foi enviado para todas as escolas do SENAI-PE, incluindo as que estão localizadas em Petrolina, Caruaru, Garanhuns, Araripina e Santa Cruz do Capibaribe, além de todas as unidades situadas em Recife.

Os questionários da pesquisa, utilizados como instrumento de coleta de dados, foram encaminhados pelo malote, através de protocolo para as doze unidades do SENAI localizadas no Recife e cidades do interior, ficando sob a responsabilidade de uma pessoa designada em cada unidade para aplicação e devolução, com prazo de entrega. O questionário era composto de três questões, duas de múltipla escolha relacionadas à questão de pesquisa e outra questão aberta para os respondentes darem sugestões à instituição sobre o tema abordado. Antes do envio das pesquisas às unidades do SENAI, foram feitos contatos internos solicitando apoio no preenchimento dos questionários e cada formulário de pesquisa foi precedido de uma carta de apresentação com os objetivos da pesquisa, mostrando a importância da participação dos respondentes. Para efeito de organização e sistematização dos dados obtidos, utilizou-se o Excel para digitação das respostas, cálculo dos percentuais e elaboração de gráficos e tabelas, cujo objetivo foi facilitar a análise e organização das informações.

A análise dos dados consistiu na organização e sistematização dos resultados da pesquisa teórico-empírica apresentando a aplicabilidade do conhecimento científico à realidade organizacional. Nesta etapa foi utilizado como instrumento de coleta de dados um questionário autoaplicável, contemplando variáveis diretamente relacionadas ao objeto de estudo. Essas variáveis foram elaboradas com foco nos objetivo geral e específicos. Para tanto, foi utilizado mais de um tipo de escala, desde a Likert até perguntas binárias. As categorias de análise foram definidas com base nas informações que fundamentaram a pesquisa e organizadas em grandes blocos de questões sobre o assunto, o que propiciou a análise de cada variável e de suas relações entre si.

A seção de discussão dos resultados relacionou os achados da pesquisa com as teorias estudadas. Os dados obtidos com a aplicação do questionário foram analisados e interpretados com o auxílio de métodos estatísticos (estatística descritiva e inferencial) e apresentados em tabelas e 
gráficos. De acordo com Patton (2002) a reincidência de termos possibilita ao pesquisador fazer a análise do conteúdo, definindo categorias e temas para a realidade estudada. Apesar de ser um processo intuitivo, segundo Merriam (1998), envolvendo a interpretação do pesquisador, tivemos o cuidado para que os resultados refletissem a visão dos entrevistados, considerando dados básicos e significativos que foram organizados com base em suas relações e regularidades. Tais procedimentos auxiliaram a sistematização das respostas em consonância com a questão de pesquisa e os objetivos apresentados no estudo, cujos resultados são apresentados na próxima seção.

\section{Resultados da Pesquisa}

Para fins de análise dos resultados, os dados são apresentados na sequência das questões levantadas:

\subsection{Ações e práticas percebidas pelos colaboradores que facilitam o desenvolvimento de uma cultura voltada à inovação e a gestão do conhecimento}

$\mathrm{Na}$ primeira pergunta do questionário, os respondentes podiam assinalar mais de uma alternativa que representasse, na sua opinião, as ações e práticas que facilitavam o desenvolvimento de uma cultura voltada à inovação e à gestão do conhecimento. Os resultados encontram-se sintetizados no Gráfico 1:

Gráfico 1 - Ações e práticas que facilitam a inovação e a gestão do conhecimento

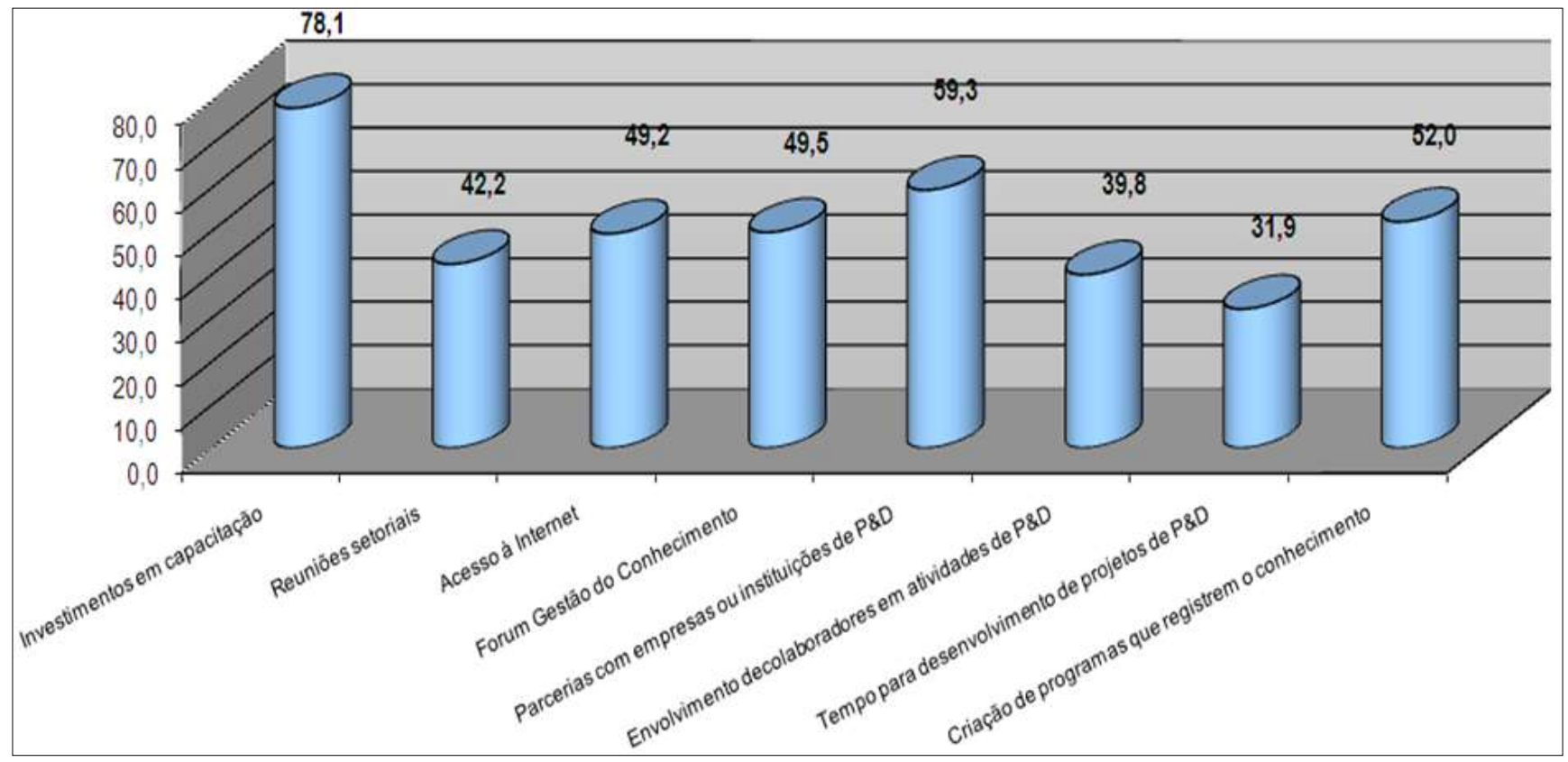

Fonte: Pesquisa de Campo (2010)

De acordo com o Gráfico 1, percebemos que todas as alternativas apresentadas tiveram um percentual representativo, porém é pertinente destacar que o item "Investimento em Capacitação" 
foi citado por $78,1 \%$ dos colaboradores, enquanto que aproximadamente metade dos respondentes também destacaram aspectos como: parcerias com empresas ou instituições de P\&D (59,3\%); criação de programas que registrem o conhecimento (52\%); a manutenção do Fórum de Gestão do Conhecimento $(49,5 \%)$; e o acesso à internet $(49,2 \%)$.

Esses resultados corroboram com os estudos de Cassapo (2006), em que este autor destaca a importância das organizações promoverem condições (investimento, práticas, políticas etc.) que estimulem a gestão do conhecimento.

O SENAI tem empreendido esforços na busca constante do desenvolvimento de uma cultura de inovação através da criação e implementação de programas e projetos inovadores promovidos pelos departamentos nacional e estadual. Um exemplo, foi à iniciativa do SENAI-PE de realizar o Fórum de Gestão do Conhecimento, que vem sendo desenvolvido desde 2007, propiciando o estímulo à criação de projetos inovadores e ao compartilhamento de conhecimentos em um ambiente favorável à pesquisa e à inovação. Existe para a realização deste fórum um planejamento de ações, dentre elas citamos a preparação de técnicos e docentes através de capacitação em elaboração de projetos de pesquisa aplicada através de uma parceria com a UFPE. Conforme Newell (2005), o conhecimento se dá na prática, através da interação e comunicação entre as pessoas. Considerando que o conhecimento é um bem intangível do indivíduo, as organizações precisam criar ambientes favoráveis à troca de experiências, à pesquisa e à inovação.

Dentre as outras ações percebidas pelos colaboradores, destacam-se as oportunidades e instrumentos para pesquisa e socialização de conhecimentos, através do acesso à internet (49,2\%), da realização de reuniões setoriais $(42,2 \%)$ e do envolvimento dos colaboradores em atividades de P\&D (39,8\%). Cassapo (2006) evidencia a importância de conectar as pessoas da organização em redes de informações, propiciando um ambiente favorável à criação e à troca de experiências. Além das ações e práticas explicitadas no questionário, outras foram citadas pelos respondentes:

- Estabelecimento de um clima que favoreça a troca de experiências e a criação de idéias;

- Criação de um programa de valorização e reconhecimento para os colaboradores envolvidos com projetos e pesquisas;

- Maior divulgação nas Escolas Técnicas dos objetivos e dos benefícios do Fórum de Gestão do Conhecimento visando atrair um maior número de participantes envolvidos em projetos;

- Formação de multiplicadores em Elaboração de Projetos de Pesquisa e Inovação;

- Maior envolvimento dos Núcleos de Informação e Documentação, disponibilizando os projetos de inovação dos colaboradores para consultas.

As informações supracitadas referem-se à categorização dos itens mais referenciados na questão aberta e refletem a percepção dos colaboradores acerca das ações e práticas essenciais ao 
desenvolvimento de uma cultura voltada à inovação e a gestão do conhecimento. É pertinente destacar que o estabelecimento de um clima que favoreça a troca de experiências e a criação de um programa de valorização e reconhecimento dessas ações, constituem aspectos importantes diretamente relacionados à cultura organizacional que, se melhor observados pela instituição, irão facilitar a aprendizagem, a inovação e a gestão do conhecimento.

\subsection{Ações e práticas percebidas pelos colaboradores que dificultam o desenvolvimento de uma cultura voltada à inovação e à gestão do conhecimento}

$\mathrm{Na}$ segunda pergunta do questionário, os respondentes também podiam assinalar mais de uma alternativa que representasse, na sua opinião, as ações e práticas que dificultavam o desenvolvimento de uma cultura voltada à inovação e à gestão do conhecimento no SENAI-PE. Os resultados encontram-se sintetizados no gráfico abaixo:

Gráfico 2 - Ações e práticas que dificultam a inovação e a gestão do conhecimento

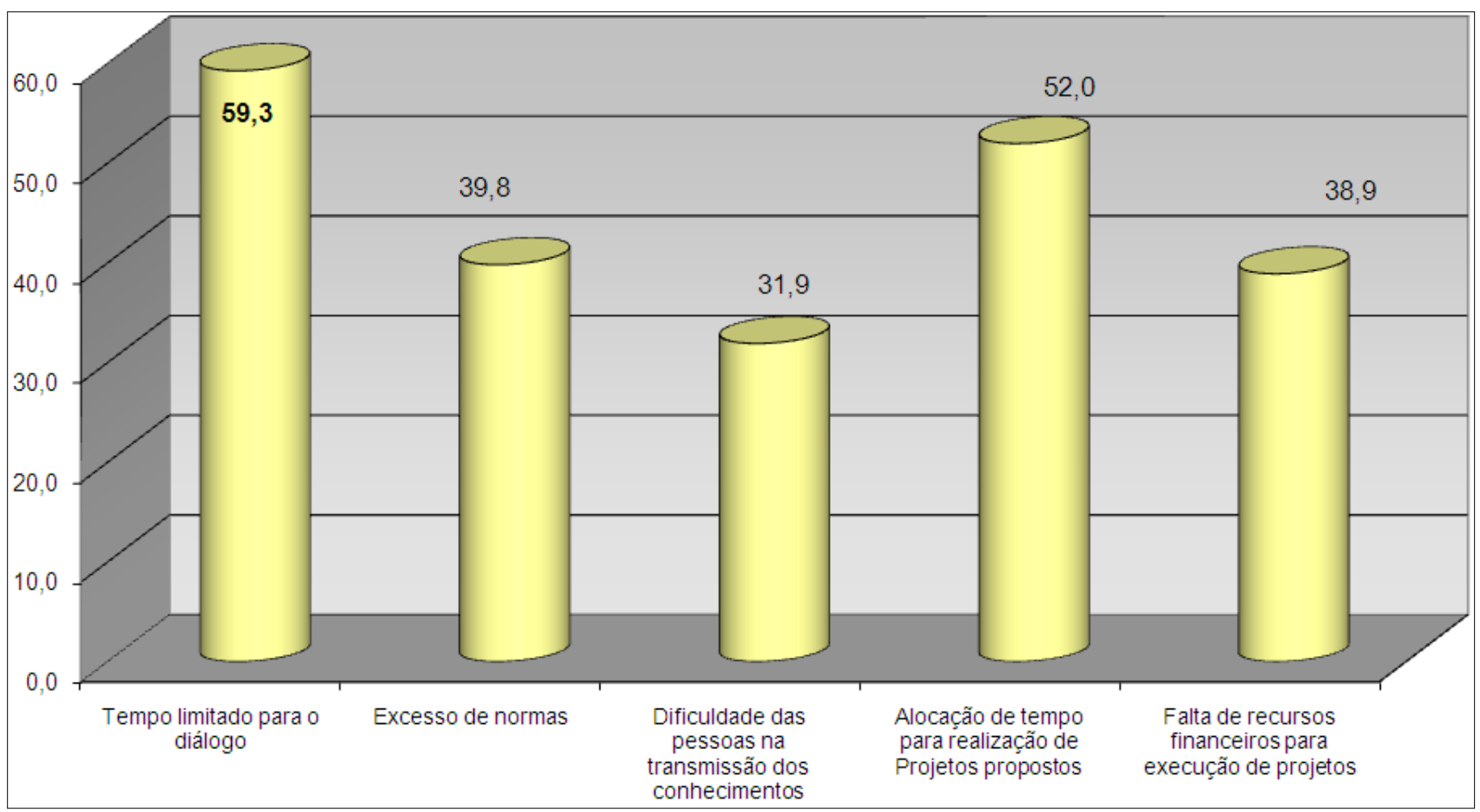

Fonte: Pesquisa de Campo (2010)

De acordo com o Gráfico 2, os resultados evidenciam que os principais obstáculos apresentados pelos respondentes estão relacionados à falta de tempo para o diálogo $(59,3 \%)$, bem como a não alocação de tempo para a realização de projetos e pesquisas (52\%). Refletir sobre a cultura da instituição torna-se de fundamental importância nesse momento, pois durante muito tempo suas ações eram focadas em Educação Profissional. Hoje com o novo desafio de desenvolver serviços técnicos e tecnológicos para as empresas, surge a necessidade de repensar novos modelos referentes a normas, procedimentos, políticas de talentos humanos e estilos de gestão. A dificuldade 
de apropriação na estatística da instituição de horas de produção para a criação de projetos de inovação é percebido como um fator limitador a criação de idéias inovadoras.

Souza et al (2008) enfatizam que existe atualmente uma lacuna no desenvolvimento da inovação nas organizações e de investimentos em pesquisa e desenvolvimento (P\&D). Considera, entretanto que esta lacuna decorre da falta de uma cultura de inovação. É preciso identificar e investir em pessoas com perfis inovadores, os chamados trabalhadores do conhecimento, pessoas autônomas, que buscam constantemente desafios, conhecimentos, e não tem receio de tentar o novo. Entretanto, a criação de uma cultura voltada para a inovação e conhecimento não se faz em curto prazo, leva tempo e este precisa ser disponibilizado para os colaboradores e reconhecido pela instituição como um fator imprescindível à aprendizagem, à inovação e, consequentemente, à gestão do conhecimento.

Dentre as outras ações e práticas dificultadoras, destacam-se o excesso de normas $(39,8 \%)$, a falta de recursos financeiros para a execução dos projetos $(38,9 \%)$ e limitações das pessoas para transmitirem seus conhecimentos $(31,9 \%)$.

Tomaél et al (2005) enfatizam que a aprendizagem organizacional é um fator preponderante para o desenvolvimento da inovação. Segundo esses autores, a cultura é um aspecto importante para o compartilhamento de informações e conhecimentos; quanto mais próximas as pessoas estiverem, mais fácil será a troca de experiências. Desenvolver a confiança reduz as barreiras para a transferência dos conhecimentos. Esse processo é altamente influenciado por fatores culturais e estruturais da empresa. Neste sentido, é imprescindível que seja desenvolvida uma cultura na instituição cujas normas não limitem a aprendizagem e a inovação, mas que as priorizem. Além disso, tal cultura precisa ser entendidas e disseminadas pelas lideranças a fim de que os recursos financeiros também sejam direcionados para esta finalidade. Alves (1997) exprime que a cultura traduz ou reflete as escolhas e preferências que foram desenvolvidas pela liderança atual ou passada da empresa e que são compartilhadas pelos seus membros. Ao mesmo tempo que as práticas que facilitam à disseminação de informações através do desenvolvimento pessoal precisam ser mais efetivas a fim de minimizar as dificuldades que os colaboradores apresentaram para "transmitir" seus conhecimentos. Além das ações e práticas explicitadas no questionário, outras foram citadas pelos respondentes: desconhecimento sobre o que realmente é e como fazer inovação tecnológica; falta de uma política que incentive o desenvolvimento de projetos de pesquisa; ausência de núcleos de pesquisa e desenvolvimento nas Escolas Técnicas do SENAI.

As informações supracitadas referem-se à categorização dos itens mais referenciados na questão aberta e refletem a percepção dos colaboradores acerca das ações e práticas que dificultam o desenvolvimento de uma cultura voltada à inovação e à gestão do conhecimento. Observamos que os colaboradores demonstram estar cientes das suas limitações ao afirmarem que desconhecem o que é e 
como fazer inovação, apresentando esse aspecto como um fator dificultador no processo de gestão do conhecimento. Por outro lado, também destacaram a necessidade de uma política voltada para o desenvolvimento de projetos, através da criação, inclusive, de núcleos de pesquisa para essa finalidade em todas as escolas.

De forma geral, a cultura perpassa todas as questões apontadas e os resultados da pesquisa teórico-empírica mostraram que a implementação de práticas inovadoras viabilizadas pela gestão do conhecimento não depende somente da vontade do indivíduo de querer aprender e compartilhar o seu conhecimento, mas principalmente de todo um conjunto de fatores estratégicos da organização, como diretrizes, sistemas, estruturas e, principalmente, de uma cultura que propicie um ambiente adequado à disseminação de informações.

\section{Conclusões e Recomendações}

Este trabalho teve como objetivo principal identificar as ações e práticas que facilitam ou dificultam o desenvolvimento de uma cultura voltada à inovação e à gestão do conhecimento no SENAI Pernambuco. A fundamentação teórica, pautada nos constructos Gestão do Conhecimento, Inovação e Cultura Organizacional, facilitou a análise dos resultados obtidos na pesquisa empírica.

A sistematização dos dados evidenciou que o fator "investimento em capacitação" foi apontado por quase $80 \%$ do total dos respondentes, como sendo uma ação que facilitava o desenvolvimento de uma cultura voltada à inovação e à gestão do conhecimento. Por outro lado, mais da metade dos colaboradores da instituição destacaram o fator tempo como um dificultador para o desenvolvimento desta cultura, por causa da falta de tempo para o diálogo, bem como a não alocação de tempo para o desenvolvimento de projetos e pesquisas. É pertinente destacar que os respondentes também puderam expor no questionário outras variáveis que achavam relevante mas que não estavam contempladas nas alternativas. Desta forma, eles apontaram como aspectos facilitadores, dentre outros, o estabelecimento de um clima que favoreça a troca de experiências e a criação de ideias e a criação de um programa de valorização e reconhecimento para os colaboradores envolvidos com projetos e pesquisas; quanto aos fatores dificultadores, os respondentes apontaram a falta de uma política que incentive o desenvolvimento de projetos de pesquisa e a ausência de núcleos de pesquisa e desenvolvimento nas escolas, ressaltando ainda que desconhecem o que realmente é e como fazer inovação tecnológica. Este último fator, provavelmente justifique porque o aspecto facilitador do investimento em capacitação foi citado por quase totalidade dos respondentes, uma vez que eles têm consciência das suas limitações acerca do tema e vêem nos programas de desenvolvimento uma oportunidade para conhecerem o assunto.

Tendo como base a bibliografia consultada foi possível identificar ações e práticas que facilitam ou dificultam o desenvolvimento de uma cultura voltada à inovação e à gestão do 
conhecimento no SENAI Pernambuco. Os resultados mostraram a cultura como uma variável fundamental para o desenvolvimento da gestão do conhecimento e inovação. Dessa forma, as questões relativas à inovação e à gestão do conhecimento precisam ser trabalhadas com foco nas questões culturais relativas à instituição.

Como ações a serem desenvolvidas constatamos a importância de a instituição orientar, capacitar e fomentar a pesquisa, acompanhar, organizar e disseminar a produção intelectual dos colaboradores (monografias, dissertações, projetos), aprimorar os serviços do núcleo de informação e documentação, aperfeiçoar a gestão da informação através de meios eletrônicos, redes de contatos bem estruturadas, criar um banco de talentos humanos para apoiar a gestão por competências, fortalecer a integração de pessoas e equipes, construir estruturas mais horizontais e flexíveis, enfim, criar uma política de gestão do conhecimento.

A relevância desse estudo deveu-se a necessidade de desenvolver no SENAI-PE uma cultura voltada à inovação e à gestão do conhecimento, objetivo este contido no Plano Estratégico, período de 2008 a 2010. A realização desse objetivo vem a contribuir fortemente para o alcance da sua missão: "Promover a educação profissional e tecnológica, a inovação e a transferência de tecnologias industriais, contribuindo para elevar a competitividade da Indústria Brasileira". Neste contexto, a instituição apresentou-se acessível aos pesquisadores para a realização deste trabalho uma vez que considera importante e válido o desenvolvimento de pesquisas científicas que possam contribuir com as suas ações. Demo (2005) destaca a importância de a pesquisa empírica ser pautada na realidade e apresentar uma "utilidade" para as organizações e para a sociedade, caso contrário, a teorização ficaria restrita ao academicismo, sem uma "aplicabilidade" crítica.

Neste contexto, este trabalho partiu de um problema com base na realidade de uma instituição e desenvolvemos uma pesquisa teórico-empírica a fim de apresentarmos uma contribuição, não apenas para a empresa, mas também para a academia. Neste âmbito, o trabalho vem cumprir um dos papéis sociais da universidade a partir da aplicabilidade da pesquisa científica à uma instituição de conhecimento que transferirá esses resultados e terá como maior beneficiária a sociedade de modo geral.

Também é pertinente apresentar algumas sugestões para pesquisas posteriores, como uma investigação do tema utilizando-se observação e entrevistas como técnicas para coleta de dados, uma vez que devido à limitação temporal para a realização deste estudo, aplicamos apenas questionários, enquanto que os demais tipos de coletas de dados favorecem um maior conhecimento da realidade estudada. Além disso, pesquisas sobre cultura contemplando a comunicação organizacional podem mostrar a coerência, ou não, entre o discurso defendido e o que é praticado por colaboradores e lideranças organizacionais, o que pode interferir de forma determinante para o desenvolvimento da gestão do conhecimento e da inovação. 
Apresenta-se, a seguir, alguns resultados da pesquisa com relação ao que poderia ser feito para o desenvolvimento de uma cultura voltada para a inovação e para a gestão do conhecimento na instituição analisada:

- Descobrir uma maneira de tornar o sistema mais leve e ágil, sair do tradicional, focar mais no que está acontecendo e enxergar as novas demandas;

- Estabelecer uma sistemática para a alocação de tempo e espaço necessários para a troca de experiências e criação de ideias inovadoras;

- Criar um programa de valorização e reconhecimento das boas ideias com prêmios e incentivos;

- Estimular a autoaprendizagem para a busca permanente do conhecimento;

- Incentivar os colaboradores a refletir e aprender com o erro, adquirindo novos conhecimentos, através do processo de reflexão-na-ação e reflexão-sobre-a-ação;

- Criar mecanismos de retenção de colaboradores talentosos;

- Aprimorar registros e armazenamento de informações e conhecimentos adquiridos ao longo dos anos para uso posterior por outros colaboradores;

- Criar e sistematizar núcleos de pesquisa e desenvolvimento nas Escolas Técnicas do SENAI-PE;

- Instituir a participação efetiva de todos os colaboradores nas tomadas de decisões;

- Vivenciar mais experiências em grupo propiciando outras aprendizagens.

Ainda, a partir dos testes estatísticos, constata-se que a inovação individual depende principalmente da existência de uma cultura organizacional e da valorização da TI, havendo um papel adicional dos gestores e da gestão do conhecimento e inovação, possivelmente como condicionantes ou canalizadores da cultura organizacional.

De acordo com as reflexões obtidas a partir da análise do SENAI-PE, pôde-se sistematizar os achados da pesquisa destacando os fatores críticos (que facilitam ou dificultam) o desenvolvimento de uma cultura voltada à inovação e à gestão do conhecimento nesta instituição, conforme ilustrado no Quadro 3: 
Quadro 3 - Fatores que facilitam/dificultam o desenvolvimento de uma cultura voltada à inovação e à gestão do conhecimento no SENAI-PE

\begin{tabular}{l} 
FATORES QUE FACILTAM \\
\hline - Valorização das pessoas e do conhecimento através de \\
investimentos significativos em programas de capacitação \\
para a aquisição e desenvolvimento de competências; \\
- Preocupação por parte de alguns gestores, de buscar \\
entender as mudanças que vêm ocorrendo e têm realizado \\
esforços no sentido de atender às novas ações demandadas \\
pelas indústrias;
\end{tabular}

- O SENAI-PE tem na sua cultura uma forma muito peculiar de se relacionar com seus colaboradores. A maioria dos gestores preza as relações de trabalho, favorecendo o diálogo e o compartilhamento de experiências e informações;

- Os colaboradores, em sua maioria, são cooperativos, têm vontade de participar e de se engajar em grupos de trabalho;

- O clima tende a ser satisfatório, apesar de que neste momento atual de grandes demandas de trabalho, os colaboradores estão se sentindo sobrecarregados e muito pressionados;

- Investimentos no desenvolvimento de projetos para participação em editais de inovação, assim como a realização de capacitações para a preparação dos colaboradores em ações voltadas a elaboração de projetos de inovação.

Fonte: Autoria própria (2011)

\section{FATORES QUE DIFICULTAM}

- Por ter um modelo de gestão muito tradicional, a instituição tende a focar-se basicamente nas ações voltadas à educação profissional tradicional. As ações para o atendimento a serviços técnicos e tecnológicos e inovação às empresas, ficam em segundo plano;

- Um grupo de gestores tem consciência dessa nova realidade e quer mudar, mas está encontrando dificuldades na passagem do modelo tradicional de gestão para um modelo de conduta orientado para a inovação;

- Baixa eficácia na gestão dos sistemas de tecnologia da informação;

- As unidades não estabelecem tempo e espaço necessários para a troca de experiências e criação de ideias inovadoras entre as equipes de trabalho;

- Falta de consciência da importância do planejamento e acompanhamento das ações nas unidades;

- A cultura da pesquisa e da elaboração de trabalhos científicos mostra-se incipiente, há poucos registros e divulgação de trabalhos.

A sistematização das respostas no Quadro 3 foi possível porque, ao segregar as respostas do questionário, observou-se similaridades de opiniões, independente do nível hierárquico ou da Unidade do SENAI-PE avaliada. Dessa forma, percebeu-se convergência de percepções por parte dos colaboradores que atuam no interior do estado ou na capital, sejam eles gestores, docentes, técnicos ou pessoal de apoio.

Esses resultados mostram a relação dos três conceitos (cultura, gestão do conhecimento e inovação) contribuindo para a melhoria dos resultados organizacionais, a partir do delineamento de ações que visem otimizar os aspectos que facilitam o alcance desses objetivos bem como minimizem os fatores críticos que limitam o desenvolvimento de uma cultura voltada à inovação e à gestão do conhecimento, seja no SENAI-PE ou em outras organizações.

\begin{abstract}
The capacity to innovate is now considered one of the most important characteristics of the organizations of excellence. In this context, it is culture as an essential element in the development of innovation and knowledge management in a company can influence positively or negatively in the process. In this light, the question that guided the development of this work was: "What are the actions and practices that facilitate or hinder the development of a culture focused on innovation and knowledge management at SENAI Pernambuco?" The theoretical and empirical the study was based on texts and research on knowledge management, innovation and organizational culture. The
\end{abstract}


research is characterized as a qualitative study, exploratory and descriptive, using a single case study. The data collection technique used was the application of an open questionnaire sent to all employees of the institution, allowing for meaningful date about the organizational culture. Among the results, it is underlined that facilitating action "investments in education and development of human talent" and hindered the action as "time factor" for both dialogue and for the preparation of projects and research at the institution. As actions to be developed, verified the importance of the institution to advise, train and promote research, monitor, organize and disseminate the intellectual output of employees (dissertations, projects), to improve the management of information through electronic media, creating a human talent pool to support the management of competencies and create a knowledge management policy.

Key-words: organizational culture, innovation, knowledge management.

\section{Referências}

ALVES, S. Revigorando a cultura da empresa: uma abordagem cultural da mudança nas organizações, na era da globalização. São Paulo: Makron Books, 1997.

ANDREASSI, T. Gestão da inovação tecnológica. São Paulo: Thomson Learning, 2007.

CANONGIA, C; SANTOS, M. M; ZACKIEWICZ, M. Foresight, inteligência competitiva e gestão do conhecimento: instrumentos para a gestão da inovação. Gestão \& Produção, v.11, n. 2, 2004.

\section{cross ref}

CASSAPO, F. Construção coletiva do saber. Disponível em: 〈http://www.conexxoes.com. br>. Acesso: 03 jun. 2006.

DEMO, P. Teoria - Para quê. Revista Eletrônica de Gestão Organizacional, UFPE, v. 3, n. 1, p. 75-85, mai/ago, 2005.

FURLANETTO, A.; OLIVEIRA, M. Fatores estratégicos para implantação de projetos de gestão do conhecimento. Revista eletrônica de gestão organizacional, v. 4, n. 4, set/dez. 2006.

GUIMARÃES, T. A.; SILVA, A. I. Conhecimento, Aprendizagem e Inovação em Organizações: uma proposta de articulação conceitual. In: XXXII Encontro Nacional dos Programas de Pós-graduação em Administração (ENANPAD). Anais eletrônicos... Rio de Janeiro: ANPAD, 2008.

HABERMAS, J. Racionalidade e Comunicação. Lisboa: Edições 70, 2002.

HAFKESBRINK, J. SCHROLL, M. Organizational Competences for Open Innovation in Small and Medium Sized Enterprises of the Digital Economy. 2010. Disponível em: <http://goo.gl/fVBSM>. Acesso em: 20 jul. 2011.

MERRIAM, S. Qualitative research and case study apllications in education. San Francisco: Jossey-Bass, 1998.

MUSSI, C. C.; ANGELONI, M. T. O compartilhamento do conhecimento no processo de implementação de sistemas de informação: um estudo de caso. In: Encontro Nacional dos Programas de pós-graduação em administração (ENANPAD). Anais eletrônicos... Curitiba, 2002.

NEWELL, S. The fallacy of Simplistic Notions of the Transfer of "Best Practice". In: BUONO, Anthony F.: POULFELT, Flemming. Challenges and issues in knowledge management. Connecticut - Information age publishing, 2005. v. 5, n. 3, p. 51-68.

NONAKA, I.; TAKEUCHI, H.; Criação do conhecimento na empresa: Como as empresas japonesas geram a dinâmica da inovação. 13. ed. Rio de Janeiro: Campus, 1997.

NOVELLI, J. G. N. O valor do valor em recursos humanos. In: Encontro Nacional dos programas de pós-graduação em administração (ENANPAD). Anais eletrônicos... Rio de Janeiro, 2003. 
OCDE. Organização para Cooperação e Desenvolvimento Econômico. Manual de Oslo: Diretrizes para Coleta e Interpretação de Dados sobre Inovação. 3. ed. Brasília: FINEP, OCDE e EUROSTAT, 1997.

PATTON, M. Qualitative research and evaluation methods. 3. ed. Thousand Oaks: Sagem, 2002.

ROBBINS, S. P. Comportamento Organizacional. 11. ed. São Paulo: Pearson Prentice Hall, 2005.

RICHARDSON, R. J. Pesquisa Social: métodos e técnicas. São Paulo: Vozes, 1989.

ROESCH, S. M. Projetos de estágio do curso de administração. São Paulo: Atlas, 1999.

SOUZA, E. C. L.; SOUZA, C. C. L . Uma Nova Maneira de Refletir os Conceitos de Cultura, Empreendedorismo e Inovação: uma metodologia de vida. In: Encontro Nacional dos Programas de pós-graduação em administração (ENANPAD). Anais eletrônicos... Rio de Janeiro, 2008.

STAKE, R. The Unique Case. In: The art of case study research. Thousand Oaks: Sabe, 1995.

STEVENS, T. Helps individuals and organizations create brilliant futures and make a difference. Disponível em <http://www.thinkleadershipideas.com>. Acesso em: 23 abr. 2011.

TOMAÉL, M. I.; ALVARÁ, A. R.; CHIARA, I. G. D. Das redes sociais à inovação. Ciência e Informação, v. 34, n. 2, mai/ago, 2005.

\section{Dados dos autores:}

Nome completo: Glória Maria Perez de Moura

Filiação institucional: Serviço Nacional de Aprendizagem Industrial (SENAI)

Departamento Regional de Pernambuco

Função ou cargo ocupado: Analista de Administração de Talentos Humanos

Endereço completo para correspondência: Rua Paulino Gomes de Souza, nº 50 apto. 602 Torre I,

Graças, Recife-PE /Brasil /CEP: 52050-250

Telefones para contato: (81) 32418871 (81) 94009977

e-mail: gperez@pe.senai.br

Nome completo: Marcos Gilson Gomes Feitosa

Filiação institucional: Universidade Federal de Pernambuco (UFPE)

Departamento: Administração

Função ou cargo ocupado: Professor Adjunto do Curso de Administração

Endereço para correspondência: Rua Professor Moraes Rego, 235 - Cidade Universitária / Recife-

PE / Brasil / CEP: 50.670-901

Telefones para contato: (81) 2126-8870 / (81) 3271-8370

e-mail: feitosam@terra.com.br

Nome completo: Fabiana Ferreira Silva

Filiação institucional: Faculdade Santa Cruz (FACRUZ) 
Departamento: Administração

Função ou cargo ocupado: Professora do Curso de Administração

Endereço para correspondência: Rua Júlia Aragão, 307 - Centro / Santa Cruz do Capibaribe-PE /

Brasil / CEP: 55.190-000

Telefones para contato: (81) 3731-4364 / (81) 9937-0501

e-mail: professorafabyana@hotmail.com

Nome completo: Flávia Andreza de Souza

Filiação institucional: Faculdades Integradas do Sertão (FIS)

Departamento: Administração

Função ou cargo ocupado: Coordenadora e Professora do Curso de Administração

Endereço para correspondência: Rua Engenheiro Vasconcelo Binttencourt, 45, Bloco Paris, Acesso 1, Apto 104, Várzea, Recife-PE, CEP: 50.740-180

Telefones para contato: (81) 3236-4923 / (81) 9706-8502

e-mail: fappita@hotmail.com

Enviado em: 27/09/2011

Aprovado em: 19/09/2012 